\title{
3-D Particle Modeling of Positive Streamer Inception from a Needle Electrode in Supercritical Nitrogen
}

\author{
Anbang Sun, Jannis Teunissen, and Ute Ebert
}

\begin{abstract}
Positive streamers need a source of free electrons ahead of them to propagate. Here we investigate a possible source of these electrons in high density media: field ionization. Zener's model is used to obtain the field ionization rate of free electrons from impurities. Including this effect, we simulate the inception of positive streamers in supercritical $\mathrm{N}_{2}$, in a needle-to-plane electrode geometry. Our 3-D particle simulation shows how the discharge branches into positive streamer channels around the electrode.
\end{abstract}

Index Terms-Positive streamers, supercritical nitrogen.

D ISCHARGES in dense media have attracted significant attention, because of their relevance to applications in high-voltage insulation/switching and chemical engineering. Different mechanisms have been proposed that could explain how dense media become ionized, such as the existence of low density regions, electron impact ionization, electroninitiated Auger ionization, photoionization, field ionization, and electrostrictive effect [1].

In this paper, we focus on positive streamer discharges in supercritical $\mathrm{N}_{2}$ at $80 \mathrm{bar}$ and $290 \mathrm{~K}$. Characteristic of positive streamers is that they need a source of free electrons ahead of them to propagate. How these free electrons are generated in supercritical $\mathrm{N}_{2}$ is still an open question. Here, we will consider the possibility of field ionization from impurities. Zener's model [2] of electron tunneling in solids is used to determine the field ionization rate, as was done in the previous studies of dielectric liquids [3], [4]. The field ionization rate $G_{I}$ is given by

$$
G_{I}(|\vec{E}|)=q^{2} n_{0} a|\vec{E}| h^{-1} \exp \left(-\pi^{2} m^{*} a \Delta^{2} / q h^{2}|\vec{E}|\right)
$$

where $q$ is the electronic charge, $h$ is Planck's constant, and $\vec{E}$ is the electric field. We assume that a density $n_{0}=10^{23} \mathrm{~m}^{-3}$ of impurities is present $(50 \mathrm{ppm})$, and that these impurities have a relatively low ionization energy of $\Delta=4.6 \mathrm{eV}$. Furthermore, we take $a=3 \times 10^{-10} \mathrm{~m}$ as

Manuscript received November 21, 2013; accepted February 24, 2014. Date of publication March 12, 2014; date of current version October 21, 2014. The work was supported by the STW under Project 12119 and Project 10118

The authors are with Centrum Wiskunde and Informatica, Amsterdam 1098XG, The Netherlands (e-mail: a.sun@cwi.nl; jannis.teunissen@cwi.nl; ute.ebert@cwi.nl).

Digital Object Identifier 10.1109/TPS.2014.2308724 the molecular separation distance and $m^{*}=9.1 \times 10^{-31} \mathrm{~kg}$ as the effective electron mass. Further investigation into these parameters is required, especially since Zener's model was developed for solids with a crystal structure.

To investigate discharges with field ionization, we have performed simulations of the inception of positive streamers in a needle-to-plane gap in supercritical $\mathrm{N}_{2}$. The simulations were carried out with a 3-D electrostatic particle-in-cell code, in which ions are assumed to be immobile and electrons are modeled as particles. Electrons randomly collide with neutral molecules that are not directly simulated but assumed to be homogeneously distributed. We include electron impact ionization, excitations, and elastic collisions, using cross sections from the SIGLO database [5]. At every time step, the self-consistent electric field is calculated.

Fig. 1(a) shows a schematic view of the computational domain. The distance from the needle tip to the plane electrode is $0.5 \mathrm{~mm}$; the radius of the needle electrode tip is $1 \mu \mathrm{m}$; the voltage applied to the needle electrode is $10 \mathrm{kV}$ and we neglect its rise time.

In Fig. 1(b), we zoom in on the area around the electrode tip, where the positive streamer discharge forms on a picosecond time scale. At $t=0 \mathrm{ps}$, no free electrons exist in the domain. After a high voltage of $10 \mathrm{kV}$ is applied to the needle electrode, free electrons and ions are dissociated from neutral molecules close to the needle electrode, due to field ionization. A bush-like streamer is first seen at $t=4 \mathrm{ps}$. This is similar to the formation of an inception cloud in atmospheric air [6]. However, in air, impact ionization and photoionization are the most important sources of free electrons, whereas in our simulations in $\mathrm{N}_{2}$ field ionization is most important. Electrons move rapidly toward the positive electrode and leave positive ions behind. This charge separation enhances the electric field near the positive ions, enabling the discharge to move forward. After $\sim 8$ ps filamentary streamers emerge, see Fig. 1(b). Tree-like branches are observed eventually, as the positive streamers repel each other. The streamers propagate at $\sim 10^{6} \mathrm{~m} / \mathrm{s}$ and have an ionization density of $\sim 10^{24} \mathrm{~m}^{-3}$.

In conclusion, field ionization might be an important source of free electrons in supercritical $\mathrm{N}_{2}$. In our 3-D particle simulations, we observe both bush-like and tree-like streamers as were observed in experiments [7]. 


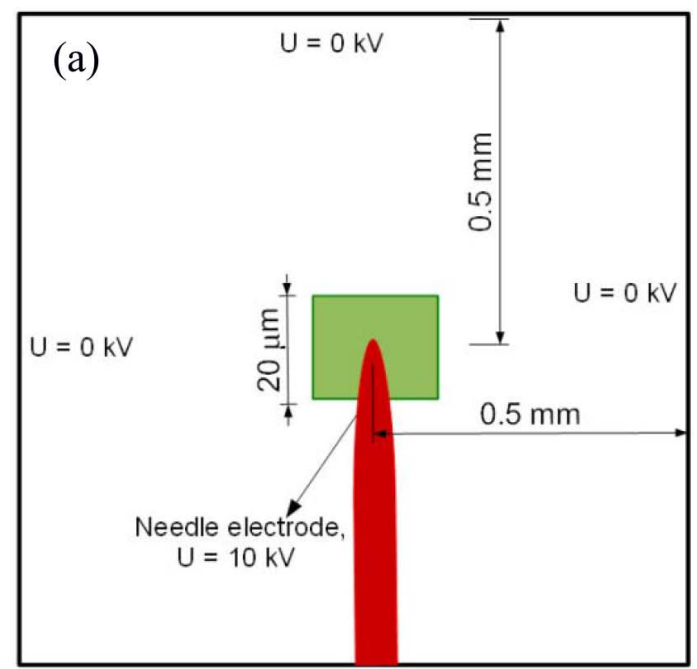

(b)

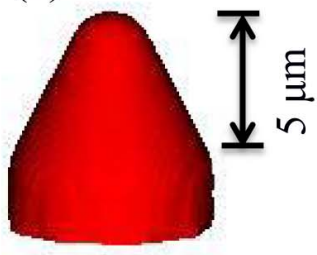

$0 \mathrm{ps}$

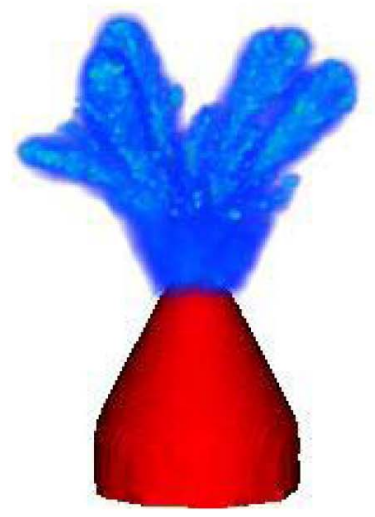

12 ps

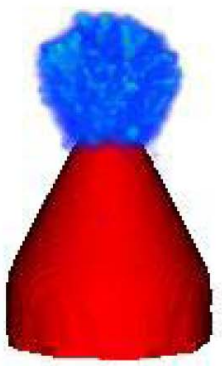

4 ps

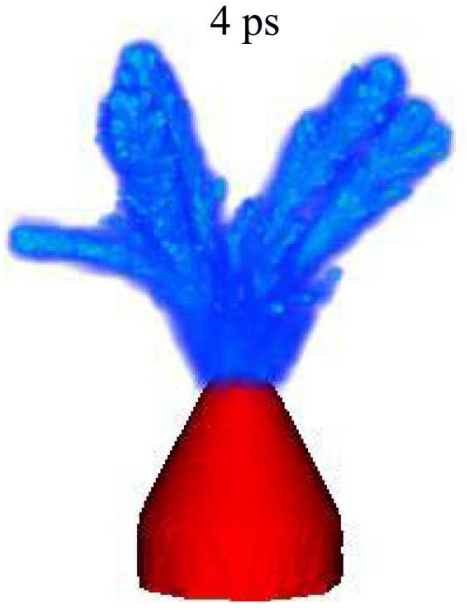

$16 \mathrm{ps}$

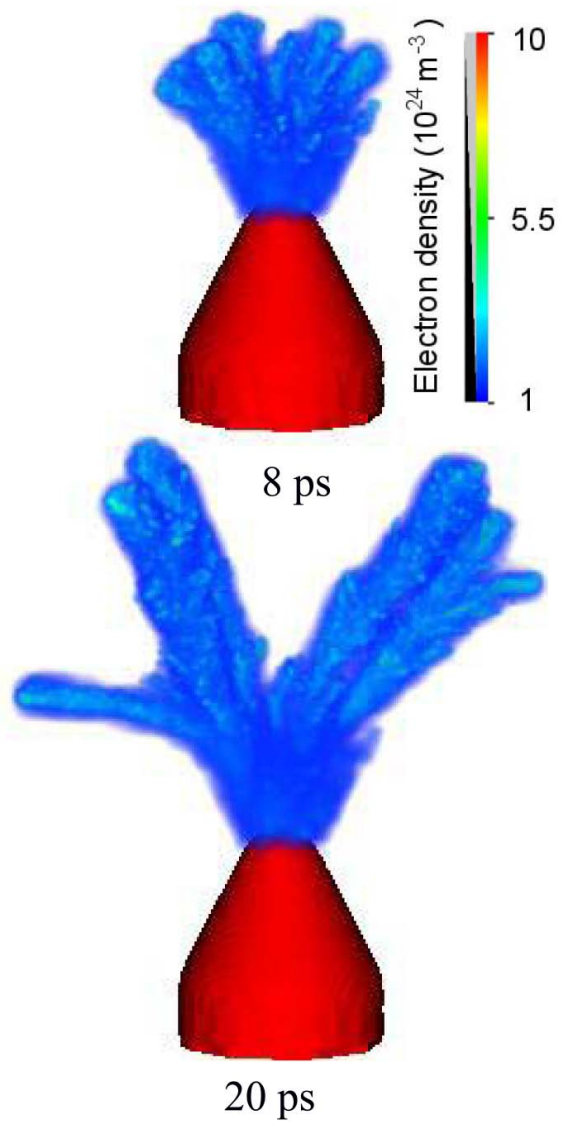

5.5

Fig. 1. (a) Whole computational domain (schematic). (b) Time evolution of the 3-D structure of the electron density. The figures are zoomed-in views of the electrode tip, indicated by the green area in (a). Medium is $\mathrm{N}_{2}$ at 80 bar and $290 \mathrm{~K}$. The figures are generated using volume rendering, and the opacity is shown next to the colorbar. Black indicates transparency.

\section{REFERENCES}

[1] J. F. Kolb, R. P. Joshi, S. Xiao, and K. H. Schoenbach, "Streamers in water and other dielectric liquids," J. Phys. D, Appl. Phys., vol. 41, no. 23, p. 234007, 2008.

[2] C. Zener, "A theory of the electrical breakdown of solid dielectrics," Proc. R. Soc. London A, Math. Phys. Sci., vol. 145, pp. 523-529, Jul. 1934.

[3] J. Jadidian, M. Zahn, N. Lavesson, O. Widlund, and K. Borg, "Effects of impulse voltage polarity, peak amplitude, and rise time on streamers initiated from a needle electrode in transformer oil," IEEE Trans. Plasma Sci., vol. 40, no. 3, pp. 909-918, Mar. 2012.
[4] J. Qian, R. P. Joshi, E. Schamiloglu, J. Gaudet, J. R. Woodworth, and J. Lehr, "Analysis of polarity effects in the electrical breakdown of liquids," J. Phys. D, Appl. Phys., vol. 39, no. 2, pp. 359-369, 2006.

[5] L. C. Pitchford and J. P. Boeuf. (2011). The Siglo Database [Online] Available: http://www.lxcat.net/

[6] T. M. P. Briels, E. M. van Veldhuizen, and U. Ebert, "Time resolved measurements of streamer inception in air," IEEE Trans. Plasma Sci., vol. 36, no. 4, pp. 908-909, Aug. 2008.

[7] P. E. Frayssines, N. Bonifaci, A. Denat, and O. Lesaint, "Streamers in liquid nitrogen: Characterization and spectroscopic determination of gaseous filament temperature and electron density," J. Phys. D, Appl. Phys., vol. 35, no. 4, pp. 369-377, 2002. 